\title{
Compliant High-precision E-Quintet Ratcheting (CHEQR) Mechanism for Safety and Arming Devices
}

John A. Kennedy

Larry L. Howell

Ihowell@byu.edu

William Greenwood

Follow this and additional works at: https://scholarsarchive.byu.edu/facpub

Part of the Mechanical Engineering Commons

\section{Original Publication Citation}

Kennedy, J.A., Howell, L.L., and Greenwood, W., "Compliant High-precision E-Quintet Ratcheting (CHEQR) Mechanism for Safety and Arming Devices," Precision Engineering, Vol. 31, No. 1, pp. 13-21, 27.

\section{BYU ScholarsArchive Citation}

Kennedy, John A.; Howell, Larry L.; and Greenwood, William, "Compliant High-precision E-Quintet Ratcheting (CHEQR) Mechanism for Safety and Arming Devices" (2007). Faculty Publications. 276. https://scholarsarchive.byu.edu/facpub/276 accepted for inclusion in Faculty Publications by an authorized administrator of BYU ScholarsArchive. For more information, please contact ellen_amatangelo@byu.edu. 


\title{
Compliant High-Precision E-Quintet Ratcheting (CHEQR) Mechanism for Safety and Arming Devices
}

\author{
John A. Kennedy \\ Dynamic Structures and Materials \\ 205 Williamson Sq. \\ Franklin, TN. 37064 \\ Larry L. Howell* \\ Department of Mechanical Engineering \\ Brigham Young University \\ Provo, UT 84602 \\ William Greenwood \\ Sandia National Laboratories \\ MS 0319, PO Box 8500 \\ Albuquerque, NM 87185
}

\begin{abstract}
Ratchet and pawl mechanisms are used in safety applications to provide mechanical isolation between inputs and an output to insure that extreme environmental conditions do not inadvertently allow an unexpected output. These devices have become smaller and are approaching a size regime where traditional precision components, such as precision bearings and springs, are not available. This paper introduces the Compliant High-precision E-Quintet Ratcheting (CHEQR) mechanism as a means of exploiting the advantages of compliant mechanisms to create safety devices that eliminate the need for bearings and springs. The pseudo-rigid-body model was used to design a mechanism with the desired force-deflection characteristics, and the result is a radical departure from traditional ratchet and pawl mechanisms. Large-scale proof-of-concept prototypes were followed by micro wire EDM fabrication of precipitation hardened stainless steel devices with flexible segment widths of $50 \mu \mathrm{m}$. The device was integrated with a $6 \mathrm{~mm}$ ratchet wheel and rotary solenoid actuator.
\end{abstract}

\section{Keywords}

Compliant Mechanisms; Safety and Arming; Pseudo-rigid-body Model; E-Quintet

\footnotetext{
*. Corresponding author. E-mail: lhowell@byu.edu, Phone: 801-422-8037, Fax: 801-422-0516
} 


\section{Introduction}

A safety and arming (S\&A) device is an electromechanical system designed to ensure protection

of personnel and equipment against premature weapon detonation through the "stockpile-to-target sequence" [1]. The "stockpile-to-target sequence" is from manufacture and storage of the weapon through weapon deployment against a target. Historically, part reliability and life of S\&A devices is 3 failures $/ 10,000$ parts per 20 years [2], but the goal for replacement designs is to have smaller devices that have 3 failures/10,000 parts per 40 years.

At the heart of many S\&A devices is a ratchet and pawl mechanism. This mechanism is designed to ratchet a mechanical wheel into the "arm" position when the predesignated electrical sequence of inputs is completed correctly. If the input sequence is incorrect, the mechanism should "lock" and render the weapon useless until it is reset. In the locked or unarmed position the S\&A device should be able to withstand extreme environments without allowing the weapon to "arm". Further descriptions can be found in [3] and [4].

As S\&A devices have become smaller and approached micro proportions, previously insignificant issues have become more influential. In the micro regime, friction is considered to be a major cause of failure. Studies have carefully quantified the effects of friction on the components of S\&A devices [5] [6]. These studies conclude that pin joints and parts that have rubbing or sliding should be avoided.

Assembly and packaging is another key aspect of micro engineering. A general rule of thumb for micro systems is that assembly can account for around $80 \%$ of the final cost of the product as well as $80 \%$ of the failures [7]. S\&A devices present an assembly and packaging challenge that is particularly complex for the following reasons [8]: 1) They cannot be fabricated monolithically because they span many processes and materials. 2) Their application requires real-time interaction with their local environment. 3) They span many orders of physical size, from sub micron structural tolerances to millimeter scale actuators and sensors. 4) They convert energy from one size scale to another. 5) They involve multiple disciplines. Thus, in order to produce a reliable and cost effective system, the assembly and packaging issues must be considered early in the design cycle [9]. 
Testing of micro S\&A device components often prove individual part reliability as well as package integrity as a whole. Common S\&A tests include: thermal cycling, high temperature aging, high voltage aging, surge testing, and shock and vibration testing [2]. Refinements and redesigns are often required before a mechanism design is accepted.

As S\&A devices have become smaller, experience has shown that the conventional spring elements, which are helical extension springs, are a major source of reliability concern. The helical springs usually have a barrel diameter of 1 to $1.5 \mathrm{~mm}$ with a wire diameter of 50 to 100 micrometers. These springs are wound on semi-automated production equipment, but the tangs or hooks on the ends of the helical barrel are generally made manually with tweezers and pliers. The highest stress in the spring is at the tangs. This high stress is exacerbated by their installation into S\&A devices where manual methods are used that can flatten or put nicks into the wire.

This paper presents a new mechanical design to replace the conventional spring elements of a ratchet and pawl mechanism with an integrated compliant mechanism. The new mechanism eliminates the assembly issues of the conventional spring elements as well as meeting the demanding design specifications for S\&A devices. It also provides an approach for making S\&A devices smaller than might otherwise be feasible using traditional components. A brief overview of the design specifications for S\&A components and relevant compliant mechanism theory will be given. The design specifications and compliant mechanism theory will then be used to detail and evaluate the new mechanism design. Finally, some initial test results and recommendations will be given.

\section{Mechanical Safety Design Specifications}

The design constraints for compliant micro ratchet and pawl mechanisms for use in high reliability applications can be generalized into the two categories of performance and manufacturing, as shown below.

\section{Performance Specifications}

Basic functionality constraints include the following:

1. The mechanism should ratchet one and only one tooth of the ratchet wheel per stroke

2. The ratchet wheel will have 36 teeth and a radius of 3 millimeters 
3. The total time to index the 36 teeth should be less than $1 \mathrm{sec}$. (ideally $0.5 \mathrm{sec}$.)

4. The mechanism actuator will be a flat rotary solenoid

5. The actuation force required by the ratchet and pawl mechanism should be significantly less than the maximum possible output force of the rotary solenoid

6. The solenoid actuator, the ratchet wheel, and the compliant, ratchet-pawl mechanism should fit on a $14 \mathrm{~mm}$ by $30 \mathrm{~mm}$ base plate, with the ratchet wheel located near the center.

7. The ratchet wheel should index on the deenergize stroke of the solenoid actuator

The following guidelines are constraints that help ensure long-term storage and life:

8. Simple designs that have minimal parts and assembly are preferred

9. Pin joints and areas of sliding friction should be minimized

10. No lubricating fluids will be considered as a part of the design

11. No materials that experience significant outgassing, degradation of material properties, or corrosion should be included in the final design [10]

\section{Manufacturing Specifications}

Micro compliant mechanism design is intimately tied to the micro manufacturing method used to make the device. Each micro manufacturing method imposes constraints on the design. Micro wire-EDM was chosen for the prototype of the ratchet and pawl mechanism introduced in this paper. Some typical constraints imposed by micro wire-EDM are:

12. Conductive materials only

13. Minimum line widths of 40 micrometers or greater

14. Tolerances of plus or minus 10 micrometers (can be reduced by adding a bias for a second run)

15. Single plane designs

The mechanism's design is affect by each of these manufacturing constraints. Precipitation hardened stainless-steel was selected for the S\&A device because it has a good ratio of yield strength to Young's modulus (ideal for compliant mechanisms) [11], is conductive, has predictable material properties, has low susceptibility to creep, and is able to operate in many harsh environments.

The minimum line width and tolerances of the micro wire-EDM process affects the mechanism compliance. The tolerance affects the in-plane flexures' movement dramatically because the inplane thickness is cubed in the area moment of inertia equation. Thus, small deviations in line thickness has a large effect on the compliant mechanism's spring constant. 


\section{Compliant Mechanisms}

Compliant mechanisms gain some or all of their motion from the flexibility of their members [11]. Many of the challenges associated with miniaturizing S\&A devices can be addressed by using compliant mechanisms.

Using compliance for motion is a characteristic of micro systems in nature. Nearly $90 \%$ of living creatures are invertebrates and the percentage of invertebrates increases as the dimensional scale decreases to the micro regime [12]. Most micro organisms use cilia and other natural forms of compliance to derive their motion. Thus, we imitate nature in designing micro mechanisms by incorporating the natural compliance of the material to achieve the mechanism's desired motion.

Some of the advantages of compliant mechanisms at the micro level include [13]: 1) They can be fabricated in a plane. 2) They require no assembly. 3) They require less space and are less complex. 4) They have less need for lubrication. 5) They have reduced friction and wear. 6) They have less clearance due to pin joints, resulting in higher precision. 7) They integrate energy storage elements (springs) with the other components. Fortunately, these advantages match many of the design specifications for S\&A devices.

Designing for compliance is challenging because the deflections are often nonlinear and, unlike rigid-body mechanisms, the motion, stresses, and forces are coupled. Many methods can be used to model large deflection mechanisms, including finite element analysis (FEA), elliptical integrals [14], topology optimization [15], and the pseudo-rigid-body model (PRBM) [11].

The PRBM allows compliant mechanisms to be modelled as rigid-body-mechanisms, thus allowing the large body of knowledge in mechanism design to be used in the design of compliant mechanisms. The PRBM is often used as a tool for initial design and visualization of compliant mechanisms that achieve complex tasks. Once the overall geometry is known, FEA can be used to verify and/or refine the design.

Pseudo-rigid-body models exist for many types of flexible segments [11] and current research is continually developing pseudo-rigid-body models for additional beam types [16]. PRBM segments that will be used in this paper include the fixed-pinned and fixed-guided segments, which are described below 


\section{Fixed-Pinned (Cantilever) Compliant Beam Segment}

The fixed-pinned compliant beam segment and its PRBM are shown in Figure 1. The flexible beam is modeled with a rigid link at the "characteristic pivot" and a torsional spring represents the resistance to motion. This model has been demonstrated to be accurate for a large range of nonlinear deflections [11]. Table 1 gives the PRBM values and explains the variables associated with the fixed-pinned segment. The variables without values are independent design choices.

\section{Fixed-Guided Compliant Beam Segment}

The fixed-guided compliant beam segment and its PRBM are shown in Figure 2. Except for the variables listed in Table 2, the variables associated with the fixed-guided beam segment are the same as the fixed-pinned beam segment shown in Table 1. The force to displace the segment $(F)$ is discussed later in this paper.

The fixed-guided and fixed-pinned compliant beam segments are building blocks and when they are used in conjunction with each other, complex mechanisms with advanced motions can be generated. The S\&A device described in this paper is such a mechanism.

\section{Concept Generation and Selection}

Given the mechanism specifications, many concepts were generated and screened. Some of the evaluation criteria used to evaluate and filter the concepts included:

- Size constraints

- Interface complexity with actuator and/or ratchet wheel

- Assembly complexity and number of components

- Analytical simplicity

- $\quad$ Sliding surfaces and pin joints (minimize possible friction failure points)

- Previous designs and work on ratcheting mechanisms

- Sensitivity to tolerance changes

The top three designs were further refined and analyzed with virtual and hardware prototypes.

The virtual prototypes were modeled with the pseudo-rigid-body model (PRBM) and finite element analysis (FEA). The virtual prototypes were then converted to functional, macro polypropylene prototypes at $20 \mathrm{X}$ scale. This quick macro prototyping using a $\mathrm{CNC}$ mill helped to evaluate issues not easily or accurately addressed with the virtual prototypes, such as areas of interference. They were also useful in evaluation possible locations and behavior of pinch points. 


\section{Compliant, High-Precision, E-Quintet Ratcheting (CHEQR) Mechanism}

After computer analysis and the evaluation of proof-of-concept prototypes, the compliant, highprecision, E-quintet ratcheting (CHEQR) mechanism was selected as the concept that best met the S\&A device specifications. A sketch of the CHEQR mechanism together with the ratchet wheel and solenoid are shown in Figure 3.

\section{Mechanism Description}

The arrows on the solenoid rotor indicate the motion that will occur about its central pin joint when the solenoid stator is energized. As the solenoid energizes, the fixed-guided flexures bend in a matching arc and the drive pawl is pulled over the next ratchet wheel tooth. The hold pawl prevents the ratchet wheel from back-rotating while the solenoid energizes.

When the solenoid deenergizes the stored energy in the fixed-guided flexures return the mechanism to its original position, indexing the ratchet wheel forward one tooth. The pinch point prevents the inertia of the ratchet wheel from over-rotating or allowing more than one tooth to ratchet at a time. Thus, each time the solenoid is energized the ratchet wheel will index one, and only one tooth.

The drive pawl runs parallel to the tooth slope to help minimize the possibility of galling or fracture when the drive pawl impacts the pinch point on the deenergize stroke. The pinch point also holds the CHEQR mechanism in a pre-stressed state so that vibration and other effects will not inadvertently index the ratchet wheel.

The solenoid rotor is balanced about its central pin-joint and pinned to the CHEQR mechanism from below. The rotor has a step at the attachment to the CHEQR mechanism so the rotor is on the same plane as the other pieces. All of the moving pieces of the system are spaced off of the base plate at their pin joints or mounting locations. This reduces the chance that they will contact the mounting plate during operation, thus eliminating possible contact surfaces where sliding friction would occur.

\section{Mechanism Manufacture and Assembly}

The design variables of the ratchet wheel, CHEQR mechanism, and rotary solenoid are shown in Figure 4 and their values are listed in Table 3 . The ratchet wheel and CHEQR mechanism are 
manufactured with 0.01 inch $(0.25 \mathrm{~mm})$ thick sheet metal using a micro wire EDM; the material is high strength precipitation hardened stainless steel (17-7 PH, Condition RH 950). The rotor and stator are manufactured from Hiperco ${ }^{\circledR}$ alloy 50 using conventional wire EDM and milling methods since ultra precision is not needed. The stator is $1.5 \mathrm{~mm}$ thick and the rotor is $1.2 \mathrm{~mm}$ thick.

The rotary solenoid is designed to deliver maximum torque with minimum friction. The Hiperco ${ }^{\circledR}$ alloy 50 metal has magnetic properties that result in excellent actuation torque characteristics. The rotary solenoid can produce a force greater than $0.07 \mathrm{~N}$ at $R_{\mathrm{sr}}$ when it is fully energized. The rotor shape is designed to include counterbalancing mass to ensure that its overall mass balances about its center pin joint. This allows it to be insensitive to non electrical actuation caused by high vibration and shock environments common to most S\&A devices.

The ratchet wheel was made from the same stainless-steel as the CHEQR mechanism with 36 evenly spaced teeth about its circumference.

The CHEQR mechanism was designed using rigid-body mechanism theory in combination with the pseudo-rigid-body model (PRBM) as described in the next section.

\section{Exploiting the E-Quintet Paradox}

The PRBM is used to determine specific geometry that exploits the E-quintet paradox to decrease the stresses and efficiently use the actuation forces. The E-quintet paradox [17] is that an E-quintet mechanism, or Assur Chain, has one degree of freedom despite the fact that the Gruebler [18] criterion predicts it to be a structure. Motion results because of the unique link geometry of the mechanism. Five links are pinned together such that they look like an upper case "E" on its side (the shaded vectors in Figure 5), thus the name E-quintet. Each of the legs of the capital letter E are then pinned to ground. The following vector equations must hold for the desired motion to occur:

$$
\begin{gathered}
\mathbf{R 2}=\mathbf{R 4}=\mathbf{R} 7 \\
\mathbf{R} \mathbf{1}=\mathbf{R 3} \\
\mathbf{R 5}=\mathbf{R 6}
\end{gathered}
$$

The CHEQR mechanism and solenoid rotor system was designed to be an E-quintet mechanism. Therefore, the characteristic radius of the CHEQR mechanism legs $(\gamma L)$ and the pin-to-pin radius 
$\left(R_{\mathrm{sr}}\right)$ on the solenoid rotor have the same deflection path. Thus, the length $(L)$ of the CHEQR mechanism and the pin-to-pin radius $\left(R_{\mathrm{sr}}\right)$ are coupled. A pin-in-slot connection between the flexure mechanism and the solonoid rotor would be an alternative to the E-quintet mechanism. This could ease some assembly and tolerance issues but would increase the friction and wear.

\section{PRBM Analysis of the CHEQR mechanism}

The CHEQR mechanism includes four compliant segments as shown in Figure 3; the two fixedguided flexures that act as the mechanism's legs and the two fixed-pinned flexures that act as the hold and drive pawls. The PRBM for the CHEQR mechanism is shown in Figure 6.

The final dimensions of the ratchet wheel, CHEQR mechanism, and solenoid rotor are listed in Table 3, and were selected to provide the desired force and deflection characteristics, while maintaining the space and stress constraints. Details on these design decisions are provided in this and following sections.

Once the basic geometry was established, CAD models of the ratcheting system taken at different points in the actuation cycle were used to determine the maximum PRBM deflection angles for each compliant segment. These angles illustrated in Figure 6 , are $\Theta_{\mathrm{dp}}=8^{\circ}, \Theta_{\mathrm{hp}}=4^{\circ}$, and $\Theta_{\max }=$ $13^{\circ}$ for the drive pawl, hold pawl, and two fixed-guided flexures respectively.

Given that the in-plane-thickness of all the compliant segments is 50 micrometers (see Table 3) and the planar thickness is $0.254 \mathrm{~mm}$, the area moment of inertia for all of the compliant segments is

$$
I=\frac{w h^{3}}{12} \approx 2.65 \mathrm{~mm}^{4}
$$

The outputs for the hold and drive pawls can be calculated using the equations in Table 1 . The resulting values are summarized in Table 4.

The yield strength $\left(\mathrm{S}_{\mathrm{y}}\right)$ for the precipitation hardened stainless-steel is approximately 1,310 MPa. Using the distortion energy theory for ductile failure, a safety factor $(S F)$ can be calculated as $S F_{\mathrm{hp}}=5$ and $S F_{\mathrm{dp}}=3.2$ for the hold pawl and drive pawl, respectively. 
The required deflection forces for the pawls are small and the safety factors are conservative. Thus, if the interface constraints of the CHEQR mechanism do not change, the bending height and length of either pawl could change considerably without yielding either pawl or affecting the operation of the mechanism.

The analysis of the CHEQR mechanism legs (see Figure 6), or fixed-guided compliant segments also shows good design flexibility. The characteristic radius $(\gamma L)$ of the fixed-guided segments can be calculated as

$$
\gamma L=6.8 \mathrm{~mm}=R_{s}
$$

where $\gamma$ is the characteristic radius factor $(\gamma)$ given in Table 1 and $L$ is listed in Table 3.

The torsional spring constant at any of the four characteristic pivots of the fixed-guided segment is given by the equation in Table 2 as

$$
K=2 \gamma K_{\Theta}\left(\frac{E I}{L}\right)=0.294 N-m m
$$

where Young's modulus $(E)$ and the stiffness coefficient $\left(K_{\Theta}\right)$ are listed in Table 1.

Recalling that the maximum necessary deflection angle for the fixed-guided legs is $\Theta_{\max }=13^{\circ}$, the maximum force $(F)$ required to deflect the mechanism can be found using the principle of virtual work as

$$
F=\frac{4 K \Theta}{\gamma L \cos \Theta}=0.04 N
$$

As shown in Table 2, the coordinate of the end of the beam $(a, b)$ can be calculated at $\Theta_{\max }=13^{\circ}$ as

$$
\begin{gathered}
a=\gamma L \sin \Theta=1.53 \mathrm{~mm} \\
b=L(1-\gamma(1-\cos \Theta))=7.83 \mathrm{~mm}
\end{gathered}
$$

where $a$ is the $x$ coordinate of the end of the deflected beam, $b$ is the $y$ coordinate.

Substituting from Table 1 and equations (4), (7), and (8), the maximum stress $\left(\sigma_{\max }\right)$ can be calculated at $\Theta_{\max }=13^{\circ}$ as 


$$
\sigma_{\max }=\frac{F a c}{2 I}=743 \mathrm{MPa}
$$

A safety factor against yielding is therefore $S F=1.8$ for a yield strength $\left(S_{\mathrm{y}}\right)$ of $1310 \mathrm{MPa}$. Theoretically, it would require more than a 70\% increase (35 micrometers) in the in-plane thickness of the fixed-guided legs $(h)$ before they would experience yielding. The tolerances held in the micro wire-EDM process are such that this concern is easily avoided.

\section{Actuation Interaction}

The dynamic analysis of micro mechanisms is challenging because electrostatic and friction forces have a far greater relative affect on functionality and dynamics than body forces. These forces are nonlinear and accurate models are under research for specific materials and situations [19].

Design specification \#3 governs ratchet time and has two parts: the energize time and the deenergize time. The energize time is primarily dictated by the input power to the rotary solenoid and how many degrees the solenoid must rotate. How many degrees the solenoid must rotate and the deenergize time are dictated by the characteristics of the CHEQR mechanism's fixed-guided legs.

The CHEQR mechanism is designed to reduce the return (deenergize) time with as small a rotation of the solenoid rotor as possible. Design criteria that bound the CHEQR mechanism are space constraints and maintaining a deflection force well below the solenoid output force. Increasing the length $(L)$ of the fixed-guided legs decreases the necessary rotation of the solenoid, however, it is coupled to the overall size of the device through the pin-to-pin radius $\left(R_{\mathrm{sr}}\right)$ of the solenoid. Thus, it is bounded by the 14 millimeters established by design specification \#6. The rotary solenoid produces a force greater than $0.07 \mathrm{~N}$ at $R_{\mathrm{sr}}$ during the energize stroke. As noted from (7), this force is almost twice the value needed to fully deflect the CHEQR mechanism. This is enough to overcome frictional effects, yet still ensures that the return time is minimized.

\section{Three Position Design}

The compliant ratchet mechanism is designed for three distinct positions: manufactured, assembled at rest (off), and cocked (on), as shown in Figure 7. The at-rest position (off) is designed so 
that some spring force is maintained on the ratchet wheel. The at-rest (off) position is designed to be at $\Theta=5^{\circ}$. Substituting into (7) yields an at-rest, or preload force of

$$
F=0.015 N
$$

This helps minimize the chance that vibration or other external effects could inadvertently ratchet the mechanism. This also means that the spring force for returning the mechanism during the deenergize stroke is greater than if the mechanism started in its manufactured position.

\section{Results and Conclusions}

The final ratchet mechanism design was manufactured and assembled at Sandia National Laboratories as shown in Figure 8. Five sheets of 0.010 inch $(0.254 \mathrm{~mm})$ thick, precipitation hardened stainless steel were simultaneously cut using the micro wire EDM. After the mechanisms were fabricated, they were analyzed to determine the actual mechanism dimensions. The first set of mechanisms were about seven to nine micrometers too small. A bias was entered into the next wire EDM run, and a second set of mechanisms was made to a tolerance of two micrometers.

Mechanisms from the second wire-EDM run were assembled and tested for functionality. An input frequency of $36 \mathrm{~Hz}$ (representing one full rotation of the ratchet wheel in 1 second) at less than 5 volts operated the CHEQR mechanism. At $72 \mathrm{~Hz}$ and 7 volts it also operated consistently with some insignificant dynamic kickback of the ratchet wheel when the drive pawl impacted the pinch point.

The CHEQR mechanism prototype meets or exceeds all the performance specifications outlined above. It consistently ratchets one and only one tooth in the specified actuation time, and the power requirements needed to ratchet in the specified time are well below the solenoid maximums. It also fits an area of 14 millimeters by 18 millimeters, which is considerably less than the size of the base plate outlined by specification \#6.

The integrated compliance of the CHEQR mechanism insured simplicity and flexibility. The conventional springs were completely removed from the design. If a rigid-body equivalent were used in its place it would require five additional links and torsional springs. Furthermore, the CHEQR mechanism uses only three pin joints and two minor places of sliding friction where the hold and drive pawls contact the ratchet wheel. 
The CHEQR mechanism also fulfills the manufacturing constraints of micro wire-EDM. The CHEQR mechanism uses only conductive materials that require no lubrication and do not experience significance degradation of their material properties. The minimum line width and tolerance specifications were met and only planar designs were needed.

If further miniaturization is desired, the minimum line width of micro wire-EDM would bound the design. Other micro manufacturing methods with smaller minimum line width specifications, such as LIGA and surface micromachining, could be considered.

Future work will be done to test and integrate the CHEQR mechanism into a fully operational S\&A device. Test devices will be built to ensure that the mechanism is reliable under severe shock, g-forces, vibrations, temperature extremes, and other harsh environments. The CHEQR mechanism will also be integrated with other S\&A components such a discriminator, coded input sequence, and chemical charges.

\section{Acknowledgements}

The assistance of Michael Cherry, Jonathan Wittwer, Daniel Wilcox, Neal Hubbard, Dennis Kuchar, Gilbert Benavides is greatly appreciated. The support of Sandia National Laboratories is also gratefully acknowledged.

\section{References}

[1] Last, H. R., Dudley B., Wood R., 1999, "MEMS Reliability, Process Monitoring and Quality Assurance," SPIE Conference on MEMS Reliability for Critical and Space Applications, Vol. 3880, Santa Clara, California, pp. 140 - 147.

[2] Archer, W. E., Sanchez, R. O., 1999, "Aging Studies for Estimating the Life of Weapon Grade Magnetic Components," Proceedings of the Electrical Insulation Conference and Electrical Manufacturing \& Coil Winding Conference, pp. 33 - 37.

[3] Kennedy, J.A., 2003, "Compliant, In-plane Ratchet and Pawl Micromechanisms for Safety Applications,” M.S. Thesis, Brigham Young University.

[4] Cannon, J.R., 2004, "Compliant Mechanisms to Perform Bearing and Spring Functions in High Precision Applications,” M.S. Thesis, Brigham Young University.

[5] Tanner, D. M., Walraven, J. A., Barnes, S. M., Smith, N.F., Bitsie, F., Swanson, S. E., 2001, "Reliability of a MEMS Torsional Ratcheting Actuator," IEEE 39th Annual International Reliability Physics Symposium, Orlando, Florida, 2001, pp. 81 - 90. 
[6] Walraven, J. A., Headley, T. J., Campbell, A. N., Tanner, D. M., 1999, "Failure Analysis of Wore Surface Micromachined Microengines," SPIE Conference on MEMS Reliability for Critical and Space Applications, Vol. 3880, Santa Clara, California, pp. 30 - 39.

[7] Beardmore, G., 1997, "Packaging for Microengineered Devices," Institution of Electrical Engineers, pp. 2/1 - 2/8.

[8] Last, H. R., Deeds, M., Garvick, D., Kavetsky, R., Sandborn, P. A., Magrab E. B., Gupta, S. K., 1999, "Nano-to-Milllimeter Scale Integrated Systems," IEEE Transactions on Components and Packaging Technology, Vol. 22, No. 2, pp. 338 -343.

[9] Deeds, M., Sandborn, P., Swaminathan, R., 2000, "Packaging of a MEMS Based Safety and Arming Device," IEEE 2000 International Society Conference on Thermal Phenomena, pp. $107-112$.

[10] Sanchez, R. O., Archer, W. E., Zich, J. L., 1997, “Application and Testing of High Temperature Materials for Solenoid Coils," Proceedings of the Electrical Insulation Conference and Electrical Manufacturing \& Coil Winding Conference, 22 - 25 Sept. 1997, pp. 325 - 332.

[11] Howell, L.L., 2001, Compliant Mechanisms, John Wiley \& Sons.

[12] Kota, S., Hetrick, J., Li, Z., Rodgers, S., Krygowsky, T., 2000, "Synthesizing High-Performance Compliant Stroke Amplification Systems for MEMS," IEEE Thirteeth Anual International Conference on Microelectromechanical Systems, 23- 27 - Jan. 2000, pp. 164 - 169.

[13] Kemeny, D.C., Magleby, S.P., and Howell, L.L., "Using Compliant Mechanisms to Improve Manufacturability in MEMS," Proceedings of the 2002 ASME Design for Manufacturability Conference, DETC2002/DFM-34178.

[14] Coulter, B. A., Miller, R. E., 1988, "Numerical Analysis of a Generalized Plane Elastica with Non-linear material Behavior," International Journal for Numerical Methods in Engineering, Vol. 26, pp. 617 - 630 .

[15] Ananthasuresh, G. K., Kota, S., 1995, “Designing Compliant Mechanisms,” Mechanical Engineering, Vol. 117, No. 11, pp. 93 - 96.

[16] Wittwer, J.W., Howell, L.L., Nov. 17, 2002, "Design of a Functionally Binary Pinned-Pinned Segment for Use as a Tansion-Compression Spring in Compliant Micro Mechanisms," IMECE 2002-33982.

[17] Norton, R. L., Design of Machinery, McGraw Hill, NewYork, New York, pp. 37.

[18] Gruebler, M., 1917, Getriebelehre, Springer Verlag, Berlin. 
[19] Knapp, J. A., de Boer, M. P., 2002, "Mechanics of Microcantilever Beams Subject to Combined Electrostatic and Adhesive Forces," IEEE Journal of Microelectromechanical Systems, Vol. 11, No. 6, pp.754-764. 


\section{Figures}

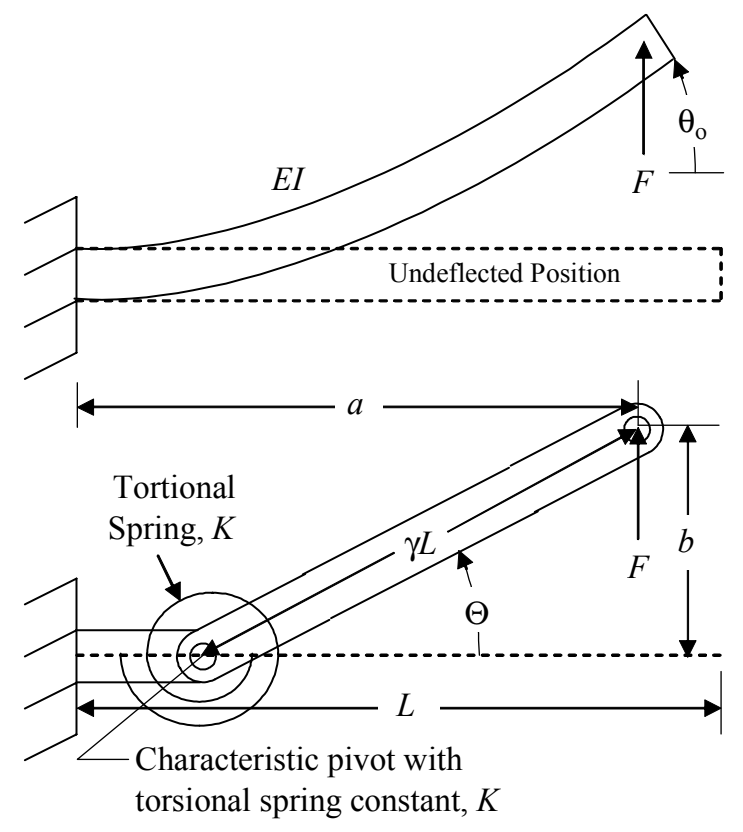

Figure 1: Fixed-pinned compliant beam segment and its PRBM 


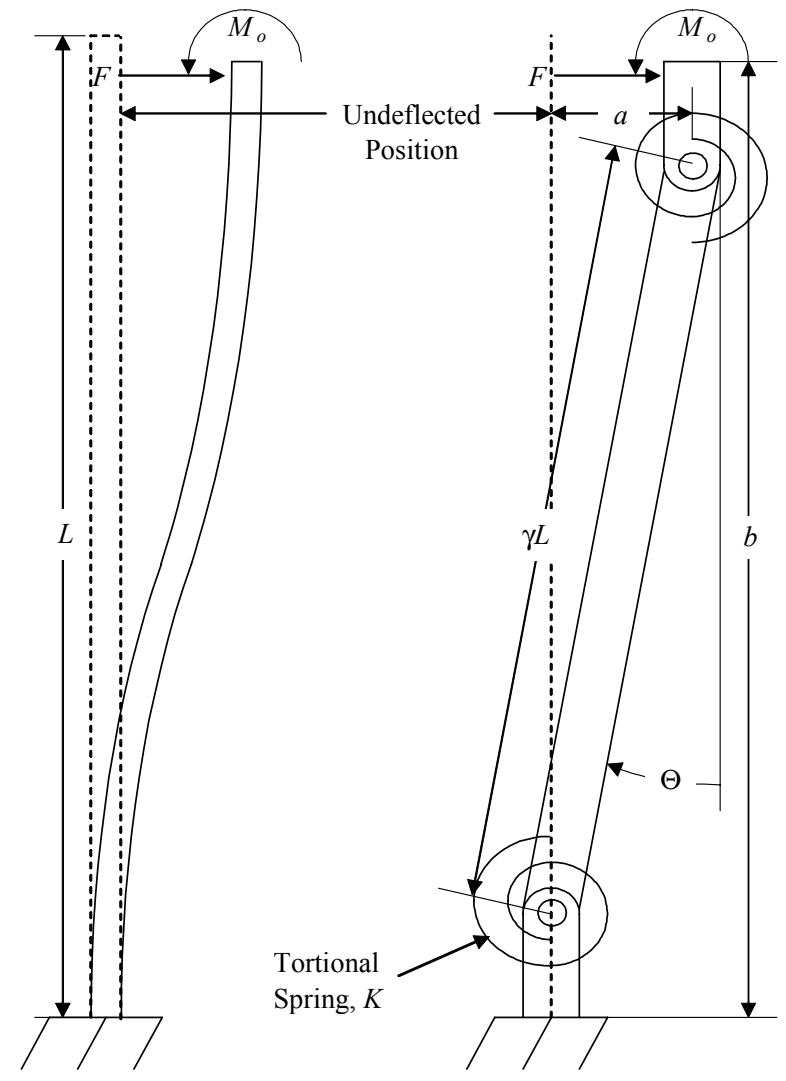

Figure 2: Fixed-guided compliant beam segment and its PRBM. 


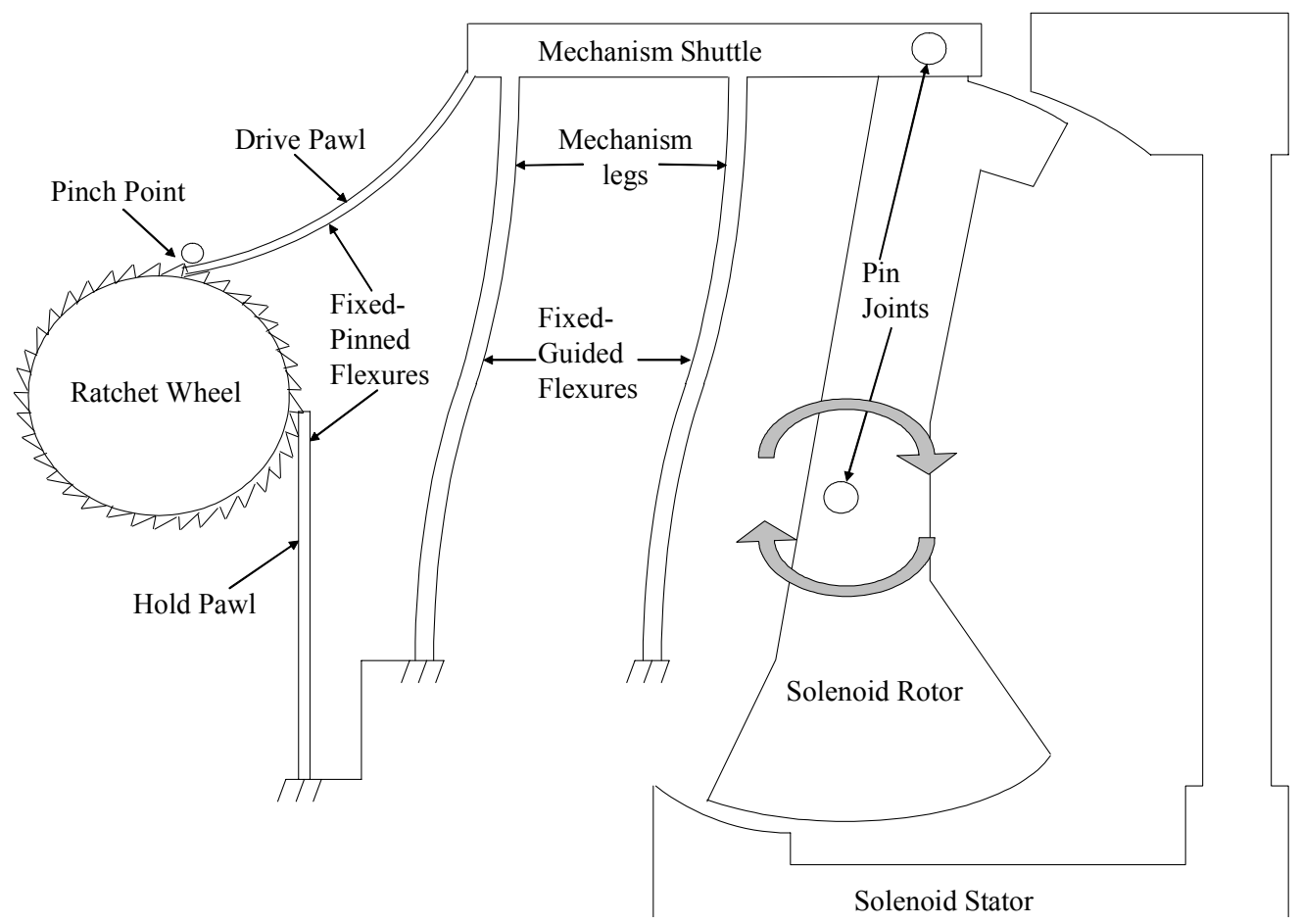

Figure 3: CHEQR mechanism with a rotary solenoid actuator 


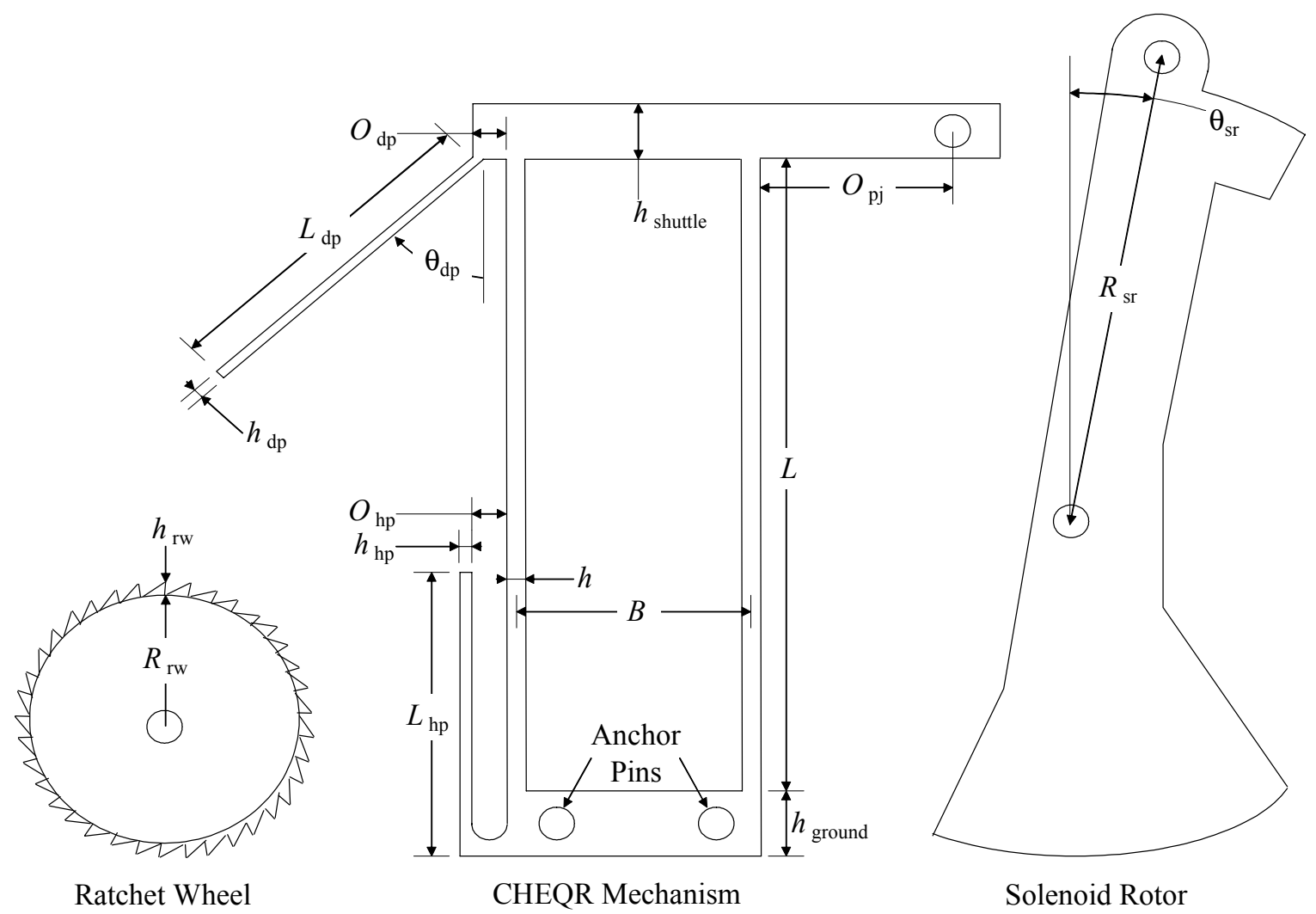

Figure 4: Manufacturing layout for the ratchet wheel, CHEQR mechanism, and solenoid rotor 


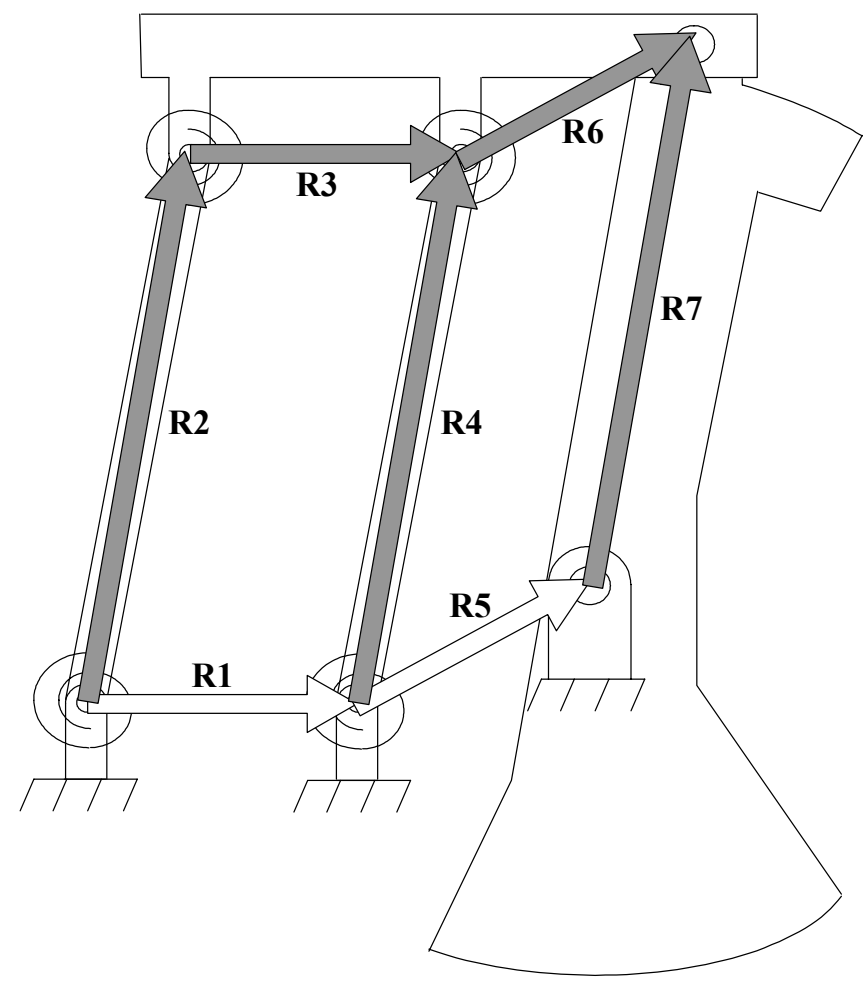

Figure 5: Linkages for an E-quintet mechanism superimposed over the CHEQR mechanism

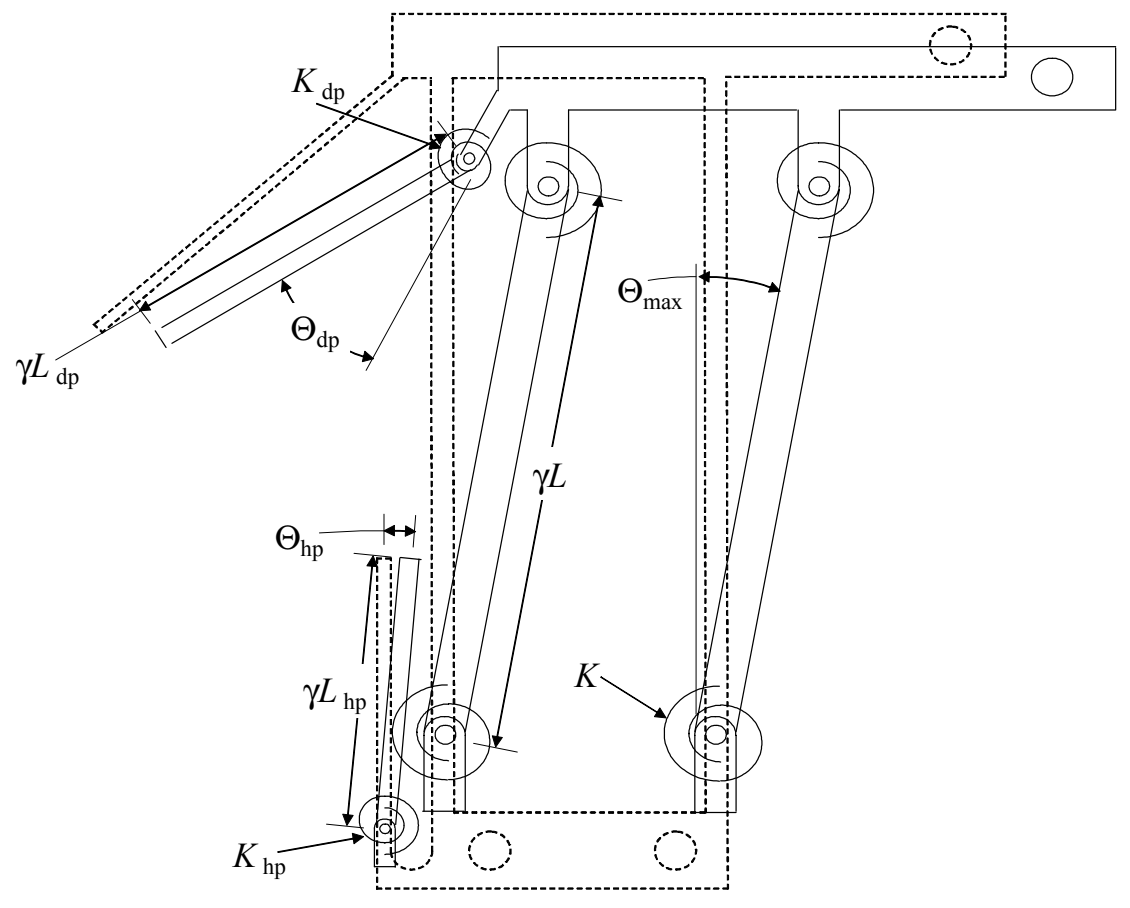

Figure 6: PRBM of the CHEQR mechanism 


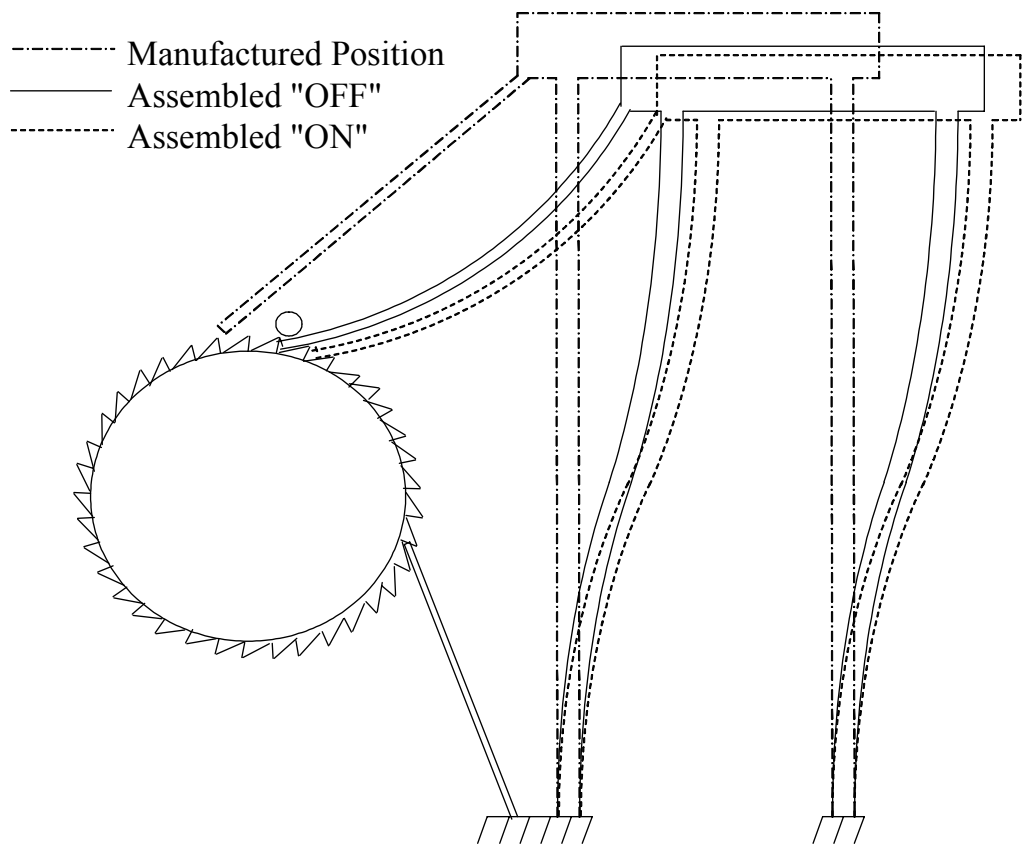

Figure 7: Three position design of the four-bar mechanism

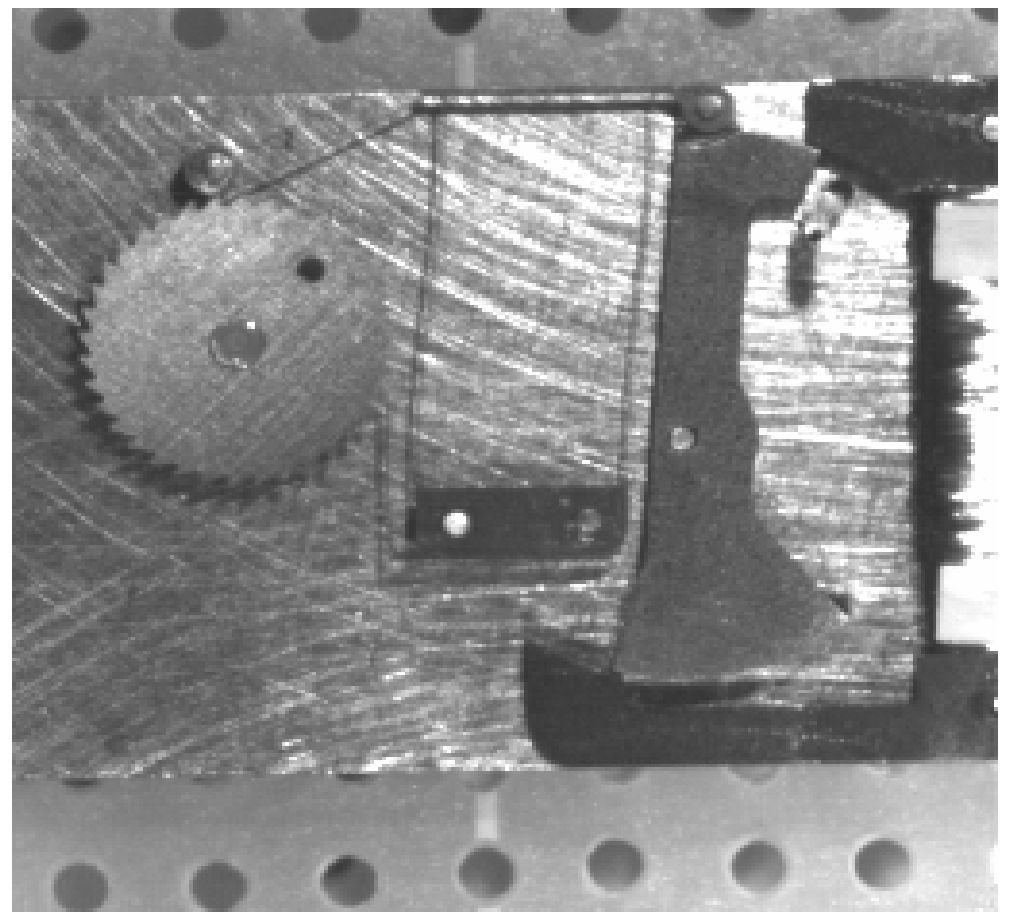

Figure 8: Scale prototype of the micro wire-EDM CHEQR mechanism. 


\section{Figure Captions}

Figure 1: Fixed-pinned compliant beam segment and its PRBM

Figure 2: Fixed-guided compliant beam segment and its PRBM.

Figure 3: CHEQR mechanism with a rotary solenoid actuator

Figure 4: Manufacturing layout for the ratchet wheel, CHEQR mechanism, and solenoid rotor

Figure 5: Linkages for an E-quintet mechanism superimposed over the CHEQR mechanism

Figure 6: PRBM of the CHEQR mechanism

Figure 7: Three position design of the four-bar mechanism

Figure 8: Scale prototype of the micro wire-EDM CHEQR mechanism. 


\section{Tables}

Table 1: PRBM variables and values for the fixed-pinned compliant beam segment

\begin{tabular}{|c|c|c|c|}
\hline & Variable & Value & Description \\
\hline \multirow[t]{3}{*}{ Inputs } & $\Theta$ & - & Pseudo-rigid-body angle \\
\hline & I & - & $\begin{array}{l}\text { Area moment of inertia (i.e. the cross } \\
\text { sectional geometry of the beam segment) }\end{array}$ \\
\hline & $L$ & - & Total length of the beam segment \\
\hline \multirow[t]{5}{*}{ Constants } & $E$ & $197 \mathrm{GPa}$ & Young's modulus of stainless steel \\
\hline & $K_{\theta}$ & 2.67 & Stiffness coefficient \\
\hline & $\gamma$ & 0.85 & Characteristic radius factor \\
\hline & $\mathrm{c}_{\theta}$ & 1.24 & Parametric angle coefficient \\
\hline & $c$ & - & $\begin{array}{l}\text { Distance from the neutral axis to the outer } \\
\text { surface of the beam }\end{array}$ \\
\hline \multirow[t]{6}{*}{ Outputs } & $\theta_{\mathrm{o}}$ & $c_{\theta} \Theta$ & End angle of beam segment \\
\hline & $K$ & $\gamma K_{\theta} E I / L$ & Spring constant for the fixed-pinned beam \\
\hline & $a$ & $L[1-\gamma(1-\cos \Theta)]$ & X coordinate of the end of the beam \\
\hline & $b$ & $\gamma L \sin \Theta$ & Y coordinate of the end of the beam \\
\hline & $F$ & $K \Theta /(\gamma L \sin (\pi / 2-\Theta))$ & Vertical force applied at the free end \\
\hline & $\sigma_{\max }$ & $\mathrm{Fac} / \mathrm{I}$ & Maximum stress of the fixed-pinned beam \\
\hline
\end{tabular}

Table 2: PRBM symbols for the fixed-guided compliant beam segment

\begin{tabular}{|l|c|c|}
\hline & Variable & Value \\
\hline \multirow{4}{*}{ Outputs } & $K$ & $2 \gamma K_{\theta} E I / L$ \\
\cline { 2 - 3 } & $a$ & $\gamma L \sin \Theta$ \\
\cline { 2 - 3 } & $b$ & $L[1-\gamma(1-\cos \Theta)]$ \\
\cline { 2 - 3 } & $F$ & - \\
\cline { 2 - 3 } & $\sigma_{\max }$ & $F a c /(2 I)$ \\
\hline
\end{tabular}


Table 3: Dimensions of the ratchet wheel, CHEQR mechanism, and the solenoid rotor.

\begin{tabular}{|c|c|c|c|}
\hline & Variable & Value (mm) & Description \\
\hline & $w$ & 0.254 & Out-of-plane thickness \\
\hline \multirow{2}{*}{$\begin{array}{l}\text { Ratchet } \\
\text { Wheel }\end{array}$} & $R_{\mathrm{rw}}$ & 3 & Radius of the ratchet wheel \\
\hline & $h_{\mathrm{rw}}$ & 0.25 & Height of the teeth of the ratchet wheel \\
\hline \multirow{6}{*}{$\begin{array}{l}\text { CHEQR } \\
\text { Mechanism }\end{array}$} & $L$ & 8 & Length of the fixed-guided flexible legs \\
\hline & $h$ & 0.05 & Bending height of the flexible legs \\
\hline & $O_{\mathrm{pj}}$ & 1 & Offset of the pin-joint \\
\hline & $B$ & 4 & Center to center distance of the legs \\
\hline & $h_{\text {shuttle }}$ & 0.2 & Height of the shuttle \\
\hline & $h_{\text {ground }}$ & 1.5 & Height of the ground link \\
\hline \multirow{4}{*}{$\begin{array}{r}\text { Drive } \\
\text { Pawl }\end{array}$} & $L_{\mathrm{dp}}$ & 4.488 & Length of the drive pawl \\
\hline & $h_{\mathrm{dp}}$ & 0.05 & Bending height of the drive pawl \\
\hline & $O_{\mathrm{dp}}$ & 0.3 & Offset of the drive pawl \\
\hline & $\theta_{\mathrm{dp}}$ & $61^{\circ}$ & Initial offset angle of the drive pawl \\
\hline \multirow{3}{*}{$\begin{array}{l}\text { Hold } \\
\text { Pawl }\end{array}$} & $L_{\mathrm{hp}}$ & 3.5 & Length of the drive pawl \\
\hline & $h_{\mathrm{hp}}$ & 0.05 & Bending height of the hold pawl \\
\hline & $O_{\mathrm{hp}}$ & 0.5 & Offset of the hold pawl \\
\hline \multirow{2}{*}{\begin{tabular}{|l} 
Solenoid \\
Rotor
\end{tabular}} & $R_{\text {sr }}$ & 8.5 & Radius of the pin to pin deflection path \\
\hline & $\theta_{\mathrm{sr}}$ & $5^{\circ}$ & Initial offset angle of the solenoid rotor \\
\hline
\end{tabular}

Table 4: Output variables for the drive and hold pawls

\begin{tabular}{|c|c|c|}
\hline Variable & Drive Pawl & Hold Pawl \\
\hline$\theta_{\max }$ & $9.92^{\circ}$ & $4.96^{\circ}$ \\
\hline$K$ & $0.264 \mathrm{~N}-\mathrm{mm}$ & $338 \mathrm{~N}-\mathrm{mm}$ \\
\hline$a$ & $4.45 \mathrm{~mm}$ & $3.49 \mathrm{~mm}$ \\
\hline$b$ & $0.53 \mathrm{~mm}$ & $0.2 \mathrm{~mm}$ \\
\hline$F$ & $10 \mu \mathrm{N}$ & $8 \mu \mathrm{N}$ \\
\hline$\sigma_{\max }$ & $410 \mathrm{MPa}$ & $262 \mathrm{MPa}$ \\
\hline
\end{tabular}

